\title{
Magnetic connection from the Earth to the solar corona, flare positions and solar energetic particle observations
}

\begin{abstract}
A. Ippolito, P. Pommois ${ }^{\star}$, G. Zimbardo, and P. Veltri
Dipartimento di Fisica, Università della Calabria, Ponte P. Bucci, cubo 31C, 87036 Arcavacata di Rende, Cosenza, Italy e-mail: pommois@fis.unical.it

Received 27 January 2005 / Accepted 3 March 2005

Abstract. Solar energetic particle (SEP) events are often associated with solar flares. Such particles propagate through the interplanetary medium, where significant levels of magnetic turbulence are found. We study the magnetic connection from the Earth to the solar corona in three dimensional magnetic turbulence. In the numerical simulation, different turbulence levels and solar wind velocities can be used. Input to the simulation is from web-based data sets, and comparison is made with the solar flare observations contained in the Goes catalogue for the years 1996, 1997, 1998, following solar minimum. For this data set, we find that SEPs can reach the Earth when the difference in the heliographic longitudes of the flare and of the magnetic foot point of the Earth is $25^{\circ}-30^{\circ}$ at most. On the other hand the longitudinal angular semi-width of the magnetic field line random walk in the solar wind, when mapped to the solar corona, is found to be typically $6^{\circ}-10^{\circ}$. The discrepancy between the two values can be explained either by the presence of a flare - associated coronal mass ejection shock, with a longitudinal semi-size of $\sim 20^{\circ}$, or by particle propagation, which could be enhanced over the field line random walk by, e.g., gyroresonant effects, or by the presence of magnetic shear between the fast and the slow streams which enhances the longitudinal spread of field lines.
\end{abstract}

Key words. solar wind - magnetic fields - turbulence - diffusion - Sun: flares

\section{Introduction}

Solar corona and/or solar wind ions are accelerated in a short time up to energies of $10 \mathrm{MeV}-1 \mathrm{GeV}$, in coronal mass ejection (CME)-driven shock waves or in strong solar flares. These particles propagate along the magnetic field lines of the solar wind and can enter in the Earth's magnetosphere, passing across the magnetopause. Enhanced fluxes of such particles represent a hazard for spacecraft operations, for navigation systems and for manned spaceflight.

Forecasting solar energetic particle (SEP) events requires a better understanding of solar flare and coronal mass ejection evolution and of energetic particle propagation in the solar wind. Indeed, considerable uncertainity remains on the details of parallel and perpendicular transport of particles, as well as on the size and location of the energetic particle source (e.g., Dalla et al. 2003; Zhang et al. 2003).

The bundle of solar wind magnetic field lines intersecting the Earth's magnetopause does not simply follow the Parker spiral, but spreads out in space because of magnetic field irregularities (Belcher \& Davis 1971; Bavassano et al. 1982). Indeed, it is well known that low frequency magnetic fluctuations induce a random walk of magnetic field lines which can be viewed as a magnetic field line diffusion (Jokipii \& Parker 1968; Rechester \& Rosenbluth 1978; Kota \& Jokipii 1995;

* Also at Center for High Performance Computing, Università della Calabria, Cosenza, Italy.
Jokipii et al. 1995; Zimbardo et al. 1995; Gray et al. 1996; Zimbardo et al. 2000; Pommois et al. 2001a). To lowest order in the drift approximation, particles follow the magnetic field lines. In such a case particle transport perpendicular to the average magnetic field is mainly due to the magnetic field structure. Further transport can be achieved by gyroresonant effects and by particles that move back and forth along the magnetic field because of parallel diffusion (Kota \& Jokipii 2000; Teufel \& Schlickeiser 2002, 2003; Matthaeus et al. 2003).

In this paper we investigate the relationships among the influence of magnetic turbulence on the magnetic connection from the solar corona to the Earth, the flare positions, and the observation of SEPs.

We perform a Monte Carlo simulation to study the magnetic connection between the Earth and the solar corona (at the level of the solar wind source surface), and compare the position of this magnetic "foot point" with that of the corresponding flare. This simulation is tailored to each specific event by using the observed values of solar wind velocity and of magnetic fluctuation level. To this end, we have analyzed web-based datasets to extract information on the flare site and class, on the solar wind velocity and magnetic turbulence level, and on the intensity of the observed SEP fluxes in the different energy bands. In order to minimize the uncertainty on the large-scale magnetic structure of the solar wind, we restrict the present analysis to the years 1996, 1997, 1998, following the solar minimum in 1995-96. Understanding particle propagation during the 
solar minimum can be a basis for the study of particle transport during the solar maximum, when a large number of high energy flares are observed. When comparing the simulation result to SEP observations, we find that the magnetic field line random walk alone cannot explain all SEP events. Possible explanations of this discrepancy, allowing for enhanced azimuthal transport or implying the presence of a shock, are discussed.

\section{Dataset from the world wide web}

A catalogue of events for 1996, 1997 and 1998 has been compiled by using experimental observations from data centers on Internet. For the flare date and time, position on the solar disc, and class, the Goes spacecraft catalogue has been used (ftp.ngdc.noaa.gov/STP/SOLAR-DATA/ SOLAR-FLARES). Only flares of class $\mathrm{C}, \mathrm{M}$, and $\mathrm{X}$ have been taken into account since strong flares are more often associated with SEPs. For the solar wind velocity and magnetic field, and for the SEP fluxes, data from the Omniweb at the National Space Science Data Center has been used (nssdc.gsfc.nasa.gov/omniweb/form/dx1.html).

At any given time, the Earth can be immersed in variablespeed stream. Clearly, we do not know the solar wind speed along the field lines that are actually magnetically connected to the Earth. Thus, we do not know what solar wind speed $V_{\text {Sw }}$ to use to determine the Parker spiral. For each event, we computed the average solar wind speed $V_{\mathrm{SW}}$ on the four days following the flare time, assuming that the statistical properties of $V_{\mathrm{SW}}$ along the Sun-Earth line resemble those along the spiral magnetic field line. In the majority of cases the deviation of the solar wind velocity with respect to the mean was less than $10 \%$. A similar averaging is made to estimate the magnetic fluctuation level $\delta B / B$ : in such a case, $B$ is averaged over ten hours, and $\delta B$ is the rms value of the fluctuation $\delta B(t)$. Also, care was taken to identify changes of the average field polarity, that is $180^{\circ}$ rotations, in order to distinguish these variations of $B$ from those due to the turbulence (Pommois et al. in preparation).

A synthesis of the relevant data is given in Table 1. The reported SEP observations come mainly from Imp8. The data time coverage is sometimes incomplete: in the table, a dash in the SEP column is entered for those events for which energetic particle data were missing after the flare.

In this paper, we pay attention mostly to the longitudinal separation between the flare and the Earth's magnetic footpoint; indeed, the non-radial structure of the solar wind magnetic field close to the Sun allows a relatively fast latitudinal transport from the flare site to the near-ecliptic plane, so that the flare latitude should have only a minor influence on the connection to Earth. On the other hand, for the cases in which SEP are accelerated by the shock of the flare-associated CME, the shock latitudinal extent is usually sufficient to cover the latitude separation from the flare site to the ecliptic (Hundhausen 1993).

Inspection of Table 1 shows, for this period after the solar minimum, that i) 6 out of $10 \mathrm{SEP}$ events are observed in connection with $\mathrm{X}$ class flares; ii) no SEPs are observed in connection with flares on the East side, even of X class; the western longitudes of the flares for which SEP are observed range from W15 (event 29) to W82 (event 40); iii) for the considered data set, enhanced fluxes of SEP are observed when the longitude separation between the flare site and the Earth magnetic foot point (computed as explained in Sect. 3) is at most $30^{\circ}$. This already gives a 30 degrees limit for the angular semi-width of either the acceleration region or the perpendicular propagation. On the other hand, there are some cases for which the longitudinal separation is substantially less than $30^{\circ}$ but no appreciable increases in the SEP fluxes were observed (events Nos. 26, 31, 33, 35, 37, 41). Possible explanations for the lack of energetic particles will be considered in the following. We also note that no strong correlation is found between the observations of SEPs and the magnetic turbulence level $\delta B / B$ or the solar wind speed $V_{\mathrm{SW}}$.

\section{Monte Carlo simulation}

The transport of the magnetic field lines in the heliosphere is evaluated with a Monte Carlo code that gives a random displacement at each step of the integration along the Parker magnetic field model. For each position on the magnetic field line, this random displacement is proportional to a "local" diffusion coefficient, which is a function of the various parameters at this particular position (e.g., fluctuation level and magnetic field correlation lengths). This code was used by Pommois et al. (2001c) to study the transport to high heliographic latitudes of particles accelerated by the co-rotating interaction region and observed by Ulysses. We summarize here the main features of the code.

The first step is to evaluate a diffusion coefficient that quantifies the local transport of magnetic field lines and takes into account both the fluctuation level and the main features of the anisotropy of the solar wind turbulence. Extensive numerical simulations of magnetic field line transport were performed by Pommois et al. (1998, 1999), Zimbardo et al. (2000), and Pommois et al. (2001a).

Since the scale of variation of the average spiral field is much larger than the correlation length of the magnetic turbulence, we can evaluate the diffusion coefficients of magnetic field lines in a local frame in Cartesian geometry. The local frame in the solar wind is so defined: the $z$ direction is along the local average interplanetary magnetic field $\boldsymbol{B}_{0}$, the $x$ direction is normal to the plane formed by the radial direction (solar wind speed direction) and $\boldsymbol{B}_{0}$, and the $y$ direction completes the right-handed system. Note that the $x$ axis points in the translatitudinal direction. The magnetic field line equations are:

$$
\frac{\mathrm{d} \boldsymbol{r}}{\mathrm{d} \xi}=\frac{\boldsymbol{B}(\boldsymbol{r})}{B_{0}}
$$

where the magnetic field is given by $\boldsymbol{B}(\boldsymbol{r})=\boldsymbol{B}_{0}+\delta \boldsymbol{B}(\boldsymbol{r})$, the average field $\boldsymbol{B}_{0}=B_{0}(\boldsymbol{r}) \hat{e}_{z}$ plus a fluctuating magnetic field $\delta \boldsymbol{B} ; \boldsymbol{r}$ is the vector position; $\xi$ is the integration parameter. The fluctuation levels relevant to the solar wind are $\delta B / B_{0} \simeq 0.5-1$; the corresponding degrees of anisotropy are $l_{x} / l_{y}=1-10, l_{z} / l_{y}=$ $1-10$, where $l_{x}, l_{y}, l_{z}$ are the turbulence correlation lengths in the $x, y$ and $z$ directions, and quantify the anisotropy of 
Table 1. List of events.

\begin{tabular}{|c|c|c|c|c|c|c|c|c|c|}
\hline$\#$ & Date & Time & Position & Class & $\begin{array}{c}V_{\mathrm{SW}} \\
\left(\mathrm{km} \mathrm{s}^{-1}\right)\end{array}$ & $\begin{array}{l}\varphi_{\text {nom }}{ }^{a} \\
\text { (deg.) }\end{array}$ & $\begin{array}{c}\Delta \varphi^{b} \\
\text { (deg.) }\end{array}$ & $\mathrm{SEP}^{c}$ & $\delta B / B^{d}$ \\
\hline 1 & $03 / 01 / 96$ & $17: 07$ & N11 W12 & $\mathrm{C}$ & 439 & 52.2 & 40 & no & 0.66 \\
\hline 2 & $22 / 04 / 96$ & $04: 43$ & S13 W76 & M & 436 & 52.5 & 24 & yes & 1.24 \\
\hline 3 & $08 / 05 / 96$ & $15: 51$ & S08 E58 & $\mathrm{C}$ & 481 & 47.6 & 105 & no & 1.92 \\
\hline 4 & $23 / 06 / 96$ & $22: 17$ & N12 E72 & $\mathrm{C}$ & 400 & 57.3 & 129 & no & 1.31 \\
\hline 5 & 09/07/96 & 09:12 & S10 W30 & $X$ & 382 & 60.0 & 29 & yes & 0.97 \\
\hline 6 & $12 / 07 / 96$ & $15: 30$ & S11 W72 & $\mathrm{C}$ & 403 & 56.8 & 16 & yes & 1.40 \\
\hline 7 & $25 / 08 / 96$ & $12: 35$ & S13 E64 & $\mathrm{C}$ & 495 & 46.3 & 110 & no & 1.11 \\
\hline 8 & $23 / 11 / 96$ & $06: 36$ & S05 E41 & $\mathrm{C}$ & 489 & 46.8 & 87 & no & 0.90 \\
\hline 9 & $29 / 11 / 96$ & $20: 42$ & S06 W47 & M & 353 & 64.9 & 17 & - & 0.64 \\
\hline 10 & $02 / 12 / 96$ & $14: 33$ & S05 W90 & $\mathrm{C}$ & 427 & 53.6 & 37 & no & 1.32 \\
\hline 11 & $19 / 12 / 96$ & $16: 10$ & S14 W09 & $\mathrm{C}$ & 419 & 54.7 & 45 & no & 0.75 \\
\hline 12 & $02 / 02 / 97$ & $06: 34$ & S23 E36 & $\mathrm{C}$ & 416 & 55.1 & 92 & no & 1.36 \\
\hline 13 & $01 / 04 / 97$ & $13: 48$ & S25 E16 & M & 459 & 49.9 & 65 & no & 1.08 \\
\hline 14 & $21 / 05 / 97$ & $20: 15$ & N05 W12 & M & 344 & 66.6 & 54 & no & 0.83 \\
\hline 15 & $29 / 06 / 97$ & $23: 52$ & N17 W82 & $\mathrm{C}$ & 411 & 55.7 & 27 & no & 1.04 \\
\hline 16 & 09/08/97 & $16: 34$ & N19 W85 & $\mathrm{C}$ & 487 & 47.0 & 38 & no & 1.76 \\
\hline 17 & $29 / 08 / 97$ & $23: 32$ & N30 E17 & M & 427 & 53.6 & 70 & no & 0.74 \\
\hline 18 & 08/09/97 & $19: 30$ & S28 W07 & M & 465 & 49.3 & 42 & no & 0.72 \\
\hline 19 & $14 / 09 / 97$ & $02: 55$ & S23 W79 & $\mathrm{C}$ & 354 & 64.7 & 15 & - & 0.48 \\
\hline 20 & $29 / 09 / 97$ & $16: 24$ & S32 W52 & $\mathrm{C}$ & 411 & 51.9 & 02 & - & 0.89 \\
\hline 21 & $04 / 11 / 97$ & $05: 58$ & S14 W33 & $X$ & 415 & 55.2 & 22 & yes & 0.82 \\
\hline 22 & $06 / 11 / 97$ & $11: 55$ & S18 W63 & $X$ & 429 & 53.4 & 10 & yes & 0.67 \\
\hline 23 & $27 / 11 / 97$ & $13: 17$ & N17 E63 & $X$ & 387 & 59.2 & 122 & no & 0.46 \\
\hline 24 & $14 / 12 / 97$ & $16: 11$ & N29 W41 & $\mathrm{C}$ & 320 & 71.6 & 30 & no & 0.57 \\
\hline 25 & $31 / 12 / 97$ & $20: 27$ & S22 W64 & $\mathrm{C}$ & 376 & 60.9 & 04 & - & 0.85 \\
\hline 26 & $01 / 01 / 98$ & 03:07 & S22 W70 & M & 365 & 62.8 & 08 & no & 1.01 \\
\hline 27 & $26 / 01 / 98$ & $22: 35$ & S17 W55 & $\mathrm{C}$ & 445 & 51.5 & 04 & yes & 1.10 \\
\hline 28 & $19 / 03 / 98$ & 01:27 & N18 E58 & M & 363 & 63.1 & 121 & no & 1.02 \\
\hline 29 & $02 / 05 / 98$ & $13: 42$ & S15 W15 & $X$ & 560 & 40.5 & 25 & yes & 0.74 \\
\hline 30 & $06 / 05 / 98$ & 08:09 & S11 W65 & $X$ & 565 & 39.8 & 26 & yes & 0.84 \\
\hline 31 & $12 / 06 / 98$ & $09: 21$ & S22 W77 & $\mathrm{C}$ & 402 & 57.0 & 21 & no & 1.50 \\
\hline 32 & $14 / 07 / 98$ & $12: 59$ & S23 E20 & M & 486 & 47.1 & 67 & no & 0.71 \\
\hline 33 & $14 / 08 / 98$ & $08: 28$ & S23 W74 & M & 341 & 67.2 & 07 & no & 0.52 \\
\hline 34 & $24 / 08 / 98$ & $22: 35$ & N35 E09 & $X$ & 616 & 37.2 & 46 & no & 0.98 \\
\hline 35 & $29 / 09 / 98$ & 02:09 & N23 W69 & $\mathrm{C}$ & 497 & 46.1 & 23 & no & 0.95 \\
\hline 36 & 07/10/98 & $17: 12$ & S20 E65 & M & 370 & 61.9 & 126 & no & 0.49 \\
\hline 37 & $18 / 10 / 98$ & $05: 49$ & N16 W57 & $\mathrm{C}$ & 533 & 43.0 & 15 & no & 0.74 \\
\hline 38 & $04 / 11 / 98$ & $12: 31$ & N16 W04 & $\mathrm{C}$ & 480 & 47.7 & 43 & no & 1.08 \\
\hline 39 & $14 / 11 / 98$ & 08:51 & N20 W63 & $\mathrm{C}$ & 442 & 53.0 & 10 & yes & 0.81 \\
\hline 40 & $22 / 11 / 98$ & $06: 33$ & S27 W82 & $X$ & 447 & 51.2 & 31 & yes & 1.80 \\
\hline 41 & $17 / 12 / 98$ & $11: 05$ & S28 W50 & $\mathrm{C}$ & 390 & 58.7 & 08 & no & 1.97 \\
\hline 42 & $28 / 12 / 98$ & $05: 48$ & N25 W27 & M & 441 & 51.9 & 25 & no & 0.79 \\
\hline
\end{tabular}

${ }^{a}$ Azimuth of the nominal magnetic footpoint of Earth in the absence of magnetic fluctuations.

${ }^{b}$ Azimuthal distance between the flare site and the Earth nominal magnetic footpoint.

${ }^{c}$ The observation or not of SEPs is indicated.

${ }^{d}$ Average of observed fluctuation level in the solar wind.

turbulence. For these cases, we have (Pommois et al. 2001a,b,c, with $i=x, y$, and $\mathcal{D}=0.030, \mu=1.52$ and $v=0.67$. 2002)

$D_{i}=\mathcal{D}\left(\frac{\delta B}{B_{0}} \frac{l_{z}}{l_{x}}\right)^{\mu} \frac{l_{x}^{2}}{l_{z}}\left(\frac{l_{i}}{l_{x}}\right)^{v}$ The dependence of the diffusion coefficient on the degree of anisotropy in the $x y$ plane $l_{y} / l_{x}$ implies that if $l_{x} \gg l_{y}$ (2) then $D_{x} \gg D_{y}$, too. Here, we trace a magnetic field line in 
the heliosphere by integrating Eq. (1); the average magnetic field $B_{0}(r)$ corresponds to the usual solar wind field in spherical coordinates $(r, \vartheta, \varphi)$ (radial distance from the Sun, latitude, azimuth)

$$
\left\{\begin{array}{l}
\left\langle B_{r}\right\rangle=B_{r \mathrm{E}}\left(\frac{r_{\mathrm{E}}}{r}\right)^{2} \\
\left\langle B_{\vartheta}\right\rangle=0 \\
\left\langle B_{\varphi}\right\rangle=-B_{r \mathrm{E}}\left(\frac{r_{\mathrm{E}}}{r}\right)^{2} \frac{\Omega r}{V_{\mathrm{SW}}} \cos \vartheta
\end{array}\right.
$$

where $B_{r \mathrm{E}}$ is the radial component of the solar wind magnetic field at the Earth, $r_{\mathrm{E}}=1 \mathrm{AU}, \Omega$ is the solar rotation rate (26 days), and $V_{\mathrm{SW}}$ is the solar wind speed. The above expression shows that when the solar wind speed $V_{\mathrm{SW}}$ is small, the Parker spiral is more tightly wound. Conversely, for large $V_{\mathrm{SW}}$, the Parker spiral is more elongated in the radial direction.

The fluctuating field component is modelled as a random process

$$
\frac{\delta B_{i}(r)}{B_{0}(r)}=\eta_{i}(\xi) A_{i}(r)
$$

where $i=x, y$ in the local frame of reference introduced above, $\eta_{x}(\xi)$ and $\eta_{y}(\xi)$ are two uncorrelated random functions and the random "force" amplitudes $A_{i}(r)$ are

$A_{i}(r)=\sqrt{6 D_{i}(r)}$.

The diffusion coefficient is obtained from Eq. (2) by using the observed level of fluctuation (see Table 1 ) and $l_{x} / l_{y}=3, l_{x}=l_{z}$, which seem to be reasonable values of the correlation lengths in the solar wind (Pommois et al. 2001c; Zimbardo et al. 2004). We note that Eqs. (2), (4), (5) imply a standard, Gaussian diffusion process. We are aware of the fact that anomalous, non-Gaussian transport regimes are possible in the solar wind (Pommois et al. 1999), and that such regimes need to be described in a different way than that corresponding to Eqs. (2), (4), (5). However, here we restrict ourselves to standard diffusion, because the order of magnitude of transverse displacement of magnetic field lines is not too different in the cases relevant to the solar wind (Zimbardo et al. 2004).

\section{Simulation results and comparison with observations}

If no magnetic turbulence is present in the solar wind, the foot point of the nominal magnetic field line passing through the Earth can easily be obtained by integrating Eq. (3) from the Earth to the solar corona. Using the average solar wind velocity, the azimuth of the nominal magnetic foot point is computed and reported in Table 1 as $\varphi_{\text {nom }}$. When magnetic turbulence is taken into account, the magnetic field line footpoint will be modified to become an area corresponding to the spread of magnetic field lines.

To describe the magnetic connection between the Earth and the solar corona, we integrate Eq. (1) for many field lines $\left(\sim 10^{5}\right)$ using the values of $\delta B / B$ reported in Table 1 , starting from the Earth's magnetopause, that is from a circle with estimated diameter of $40 R_{\mathrm{E}}$, with $R_{\mathrm{E}}$ being the Earth's radius, and reaching the solar wind source surface at $r \sim 3 R_{\odot}$.
We then show the contour levels of the distribution of field lines from the Earth to the arrival points at the solar wind source surface. The contour levels represent the probability density of magnetic connection with the Earth, as obtained from the Monte Carlo simulation, with isolevels spaced by a factor of 2. The magnetic field line positions are plotted in heliographic latitude and longitude, on the background of the magnetic field maps at the source surface of the solar wind, as computed by the Wilcox Solar Observatory at $r=2.5 R_{\odot}$ (http://sun.stanford.edu/ wso/wso.html), for the period relevant to the considered flare. For each event, the actual turbulence level $\delta B / B$, reported in Table 1 was used.

The top panels of the figures show the relative position of the flare site and the "foot point" area for sample events, chosen to represent different possible cases. The energetic particles time profile for energies $E>1 \mathrm{MeV}, E>10 \mathrm{MeV}$, $E>60 \mathrm{MeV}$, and the solar wind speed from Imp8 are shown in the middle and lower panels of the figures for the same events. The vertical dashed line in the lower panels indicates the flare time. As can be seen from Fig. 1, the contours representing the magnetic foot point are slightly elongated in the latitude direction, given that $l_{x}>l_{y}$ implies fast latitudinal transport (see Eq. (2) and Pommois et al. 2001c). Comparing the top panels of the figures, it can be seen that the width of the "foot point" area grows with the magnetic fluctuation level, as expected: this is apparent when we compare Fig. 1 (event 22), for which $\delta B / B \simeq 0.67$, Fig. 2 (event 26), for which $\delta B / B \simeq 1$, and Fig. 4 (event 40 ), for which $\delta B / B \simeq 1.8$. These figures allow us to evaluate the extent of the propagation due to field line random walk: considering the semi-width at half-height of the probability density, corresponding to the first contour in the figures, this yields about $\pm 6^{\circ}-10^{\circ}$ in longitude, and about $\pm 10^{\circ}-15^{\circ}$ in latitude (the exact value depending on the turbulence level).

For the individual cases, we show in Fig. 1 the data for event 22, which exhibited strong SEP intensity increases soon after the flare. In this case the area corresponding to the Earth magnetic foot point is rather close to the flare site. This event shows the typical time profiles for gradual events in the western hemisphere, which are thought to be associated with a shock wave travelling in the solar wind (e. g., Cane et al. 1998; Reames 1999). Figure 2 shows event 26, for which no particle flux increase was detected after the flare, in spite of the fact that the nominal magnetic foot point of the Earth is close to the flare, $\Delta \varphi \simeq 8$, see the Table 1 . On the other hand, as can be seen from Fig. 2, top panel, the Earth's magnetic foot area and the flare lie on opposite sides of the magnetic equator. This implies that a sector boundary is found between the flare and the Earth, and it is well known that sector boundaries are an obstacle to particle propagation (Kallenrode 1993). Indeed, at a sector boundary the average magnetic field direction changes from sunward to antisunward, or vice versa, causing a strong deflection in particle propagation. Also, for this event we have an $\mathrm{M}$ class flare, which, arguably, is a weaker energetic particle source than $\mathrm{X}$ class flares. The presence of sectory boundaries and/or a $\mathrm{C}$ or $\mathrm{M}$ class flare can explain why no particles are observed for events $31,33,35,37,41$, for which $\Delta \varphi$ is $23^{\circ}$ at most. It could be argued that the acceleration region for $\mathrm{C}$ and $\mathrm{M}$ class flares is smaller than for $\mathrm{X}$ class flares, which are more 

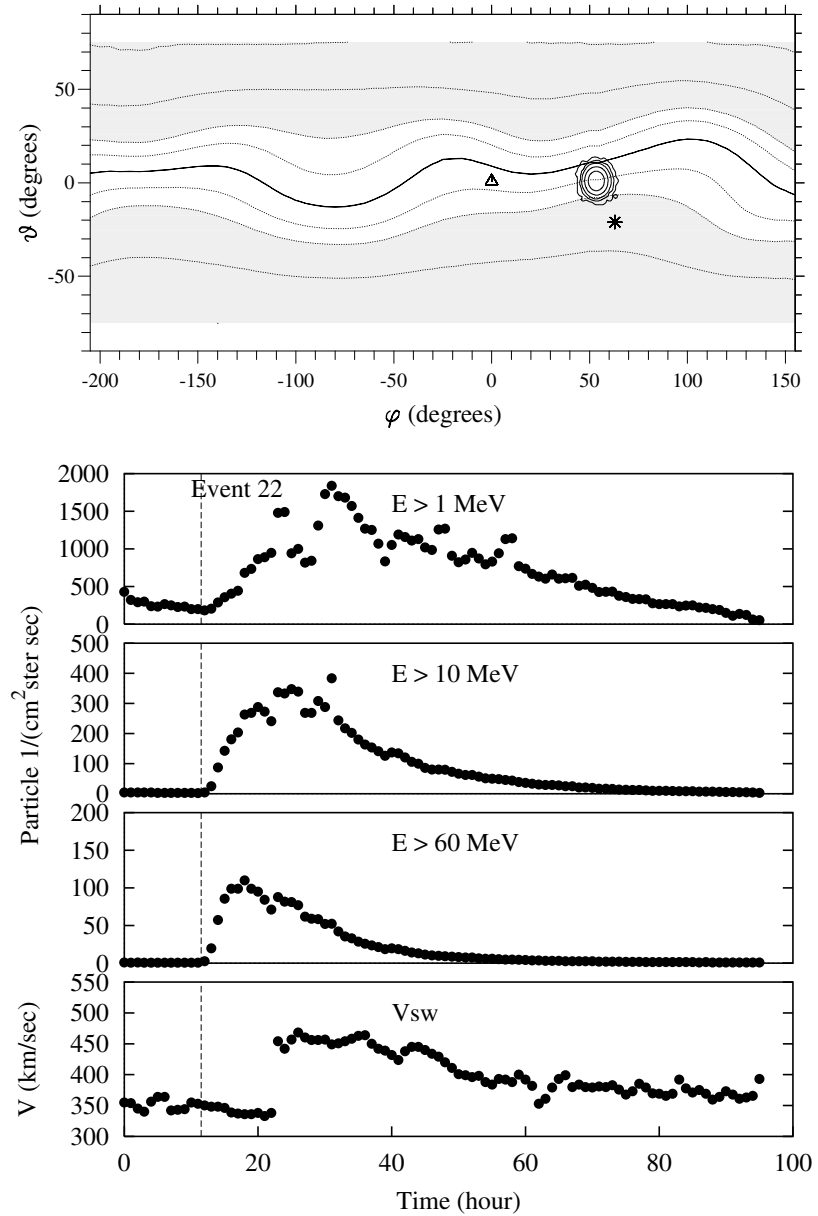

Fig. 1. Top panel: relative position of the Earth (triangle), of the flare site (asterisk) and of the Earth's magnetic foot area (solid contour levels) projected on the solar magnetic configuration at the source surface $\left(r=2.5 R_{\odot}\right.$ ) magnetograms from the Wilcox Solar Observatory. The thick solid line represents the magnetic equator and the dotted lines the isointensity contours of $|\boldsymbol{B}|$ for event 22 on Nov. 6, 1997. Lower panels: energetic particle time profiles and solar wind speed (hourly averaged data) for event 22 . Time is in hours after the beginning of the event day. The vertical dashed line correspond to the flare time.

likely to be associated with a shock. We also note that a small but non-negligible number of small-size CMEs are found in the study of Hundhausen (1993). If one assumes that $C$ and $M$ class flares could be associated with small-size shocks, it can be understood why SEP are not observed.

Figure 3 shows an event for which SEPs were observed, with the flare in a rather central position on the solar disc, $\varphi \simeq 15 \mathrm{~W}$. During the flare period the solar wind speed was high, $V_{\mathrm{SW}} \simeq 600 \mathrm{~km} \mathrm{~s}^{-1}$, see bottom panel of Fig. 3, so that the Parker spiral was rather elongated, and the magnetic foot area is located at $40 \mathrm{~W}$. As shown by the top panel of Fig. 3, the Earth, the flare and the "magnetic connection" were all in the same sector, so that particles are not influenced by the need to cross a sector boundary. As a consequence, clear particle enhancements are observed in the three of the considered energy bands (although not as strong as for event 22). A pair of gaps in the measured energetic particle profiles can be seen, as discussed in Sect. 2.
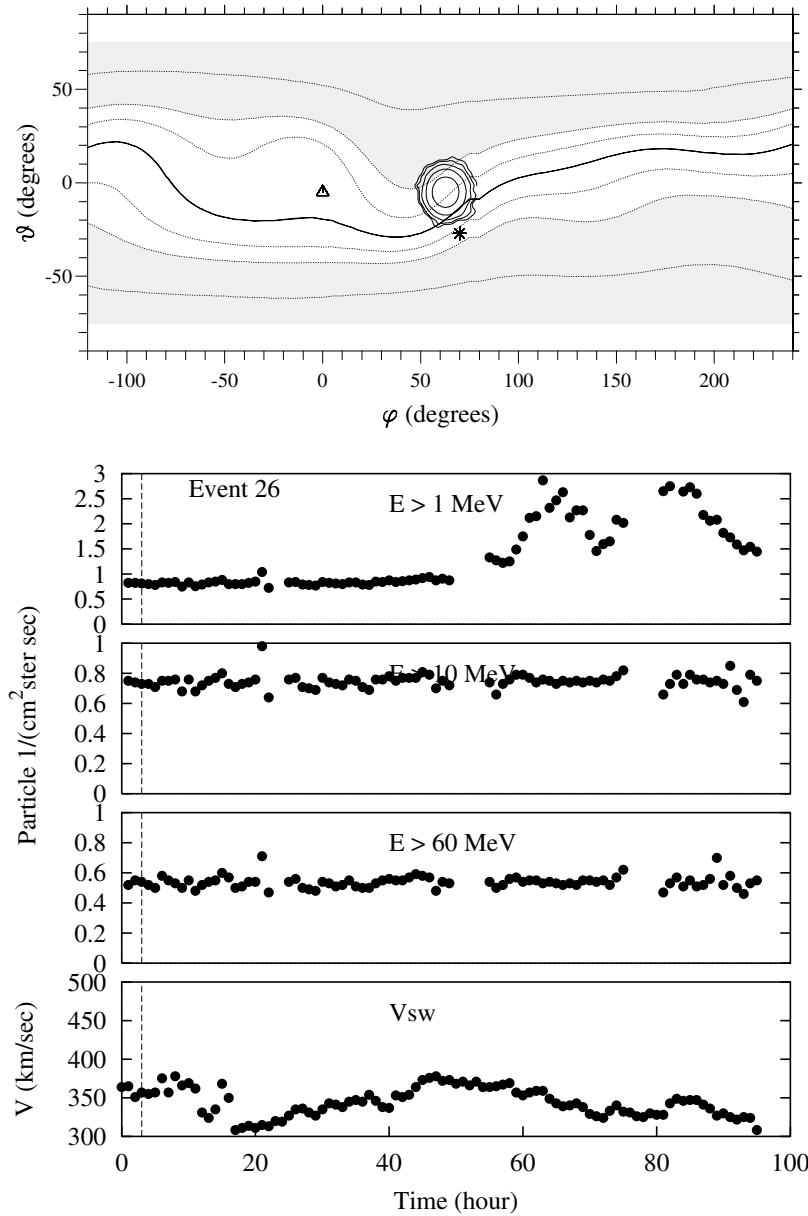

Fig. 2. Same as Fig. 1, but for event 26, on Jan. 1, 1998.

Figure 4 shows the data and the simulation result for event 40 , for which $\Delta \varphi=31^{\circ}$ (see Table 1). Particle enhancements are observed in the three energy bands. The weakness of the signals can be related to the fact that $\Delta \varphi=31^{\circ}$, that is to the fact that the separation between the flare site and the nominal magnetic foot point is at a maximum (for this data set). This means that the overlap between the magnetic flux tubes connected to the Earth and the region populated by energetic particles is at a minimum. We also notice that the magnetic structure of the solar wind source surface is rather complex, given that event 40 occured on November 22, 1998, that is in a period of quickly growing solar activity, and that the magnetic foot point area is on a different magnetic sector than the Earth. This implies that the present numerical simulation is not reliable in such cases, since considerable distortion of the Parker magnetic field is found when crossing the heliospheric current sheet. This difficulty motivates our choice to study only the period following the solar minimum.

\section{Discussion and conclusions}

The data analysis and the numerical simulations reported above have given us the following result: for the considered data set, we find that SEPs can reach the Earth when the difference in the heliographic longitudes of the flare and of the nominal magnetic foot point of the Earth is $25^{\circ}-30^{\circ}$ at most. In this data 

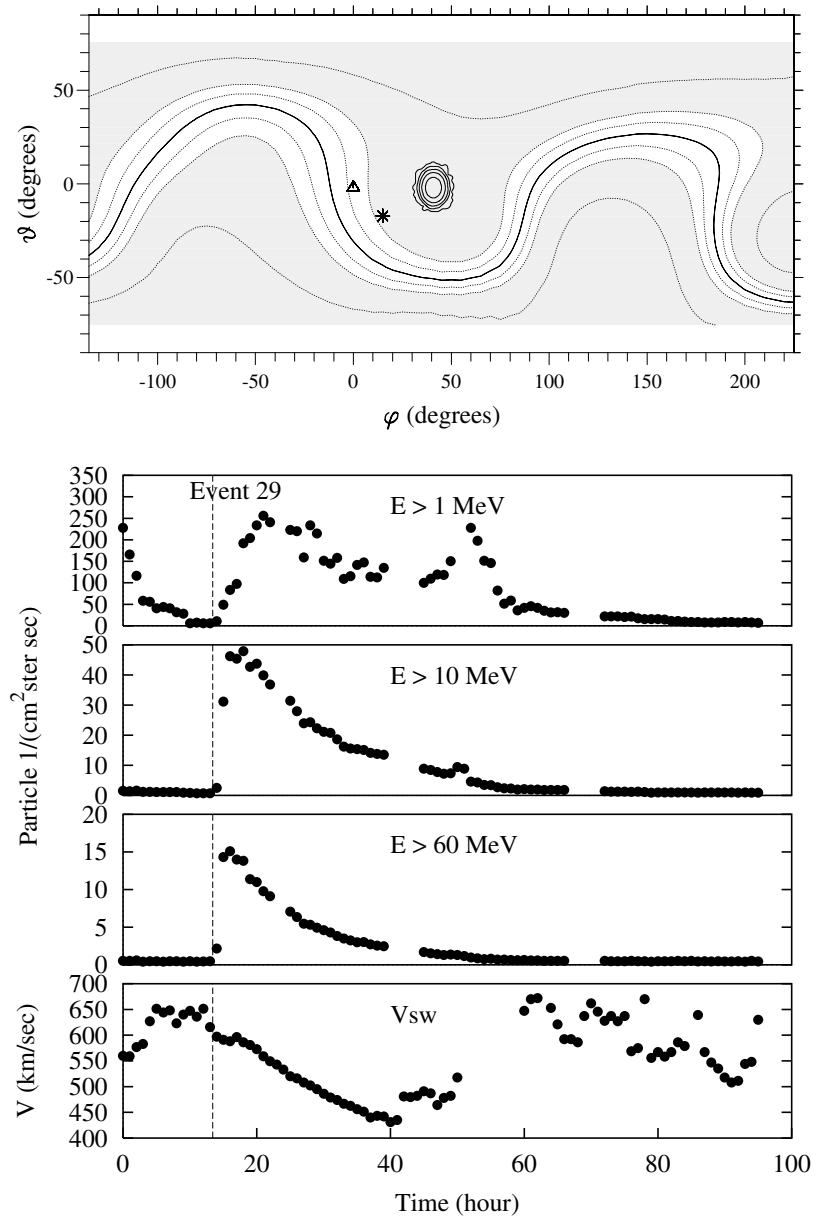

Fig. 3. Same as Fig. 1, but for event 29, on May 2, 1998.

set, no significant energetic particles fluxes are observed in association with flares from the East longitudes. From the simulation of field line random walk, we find that the azimuthal semi-width of the field lines going from the Earth's magnetosphere to the solar corona is $6^{\circ}-10^{\circ}$. Clearly, field line random walk alone is not enough to explain the detection of energetic particles on the Earth when the nominal magnetic foot point is far from the flare. Among possible explanations, we consider (i) the presence of a flare-associated CME shock, (ii) particle longitudinal transport in addition to field line transport, (iii) the effect of magnetic shear in the Parker spiral field due to the presence of fast and slow streams.

The presence of a shock accelerating particles, expecially after strong flares, constitutes the "current paradigm" for gradual SEP events (Reames 1999; Dalla et al. 2003). In such a case, the result of a $6^{\circ}-10^{\circ}$ longitudinal width for the field line random walk, together with the finding, reported above, that SEPs are observed on Earth when the azimuthal separation between the flare and the nominal foot point is at most $30^{\circ}$, gives an estimate maximum angular (azimuthal) half-size for the particle acceleration region of about $20^{\circ}-24^{\circ}$. This is consistent with previous estimates of the average sizes of CME shocks of $47^{\circ}$ (Hundhausen 1993), which were obtained by analysis of the images of the coronograph/polarimeter flown on the SMM spacecraft. This also agrees, as a typical size, with the
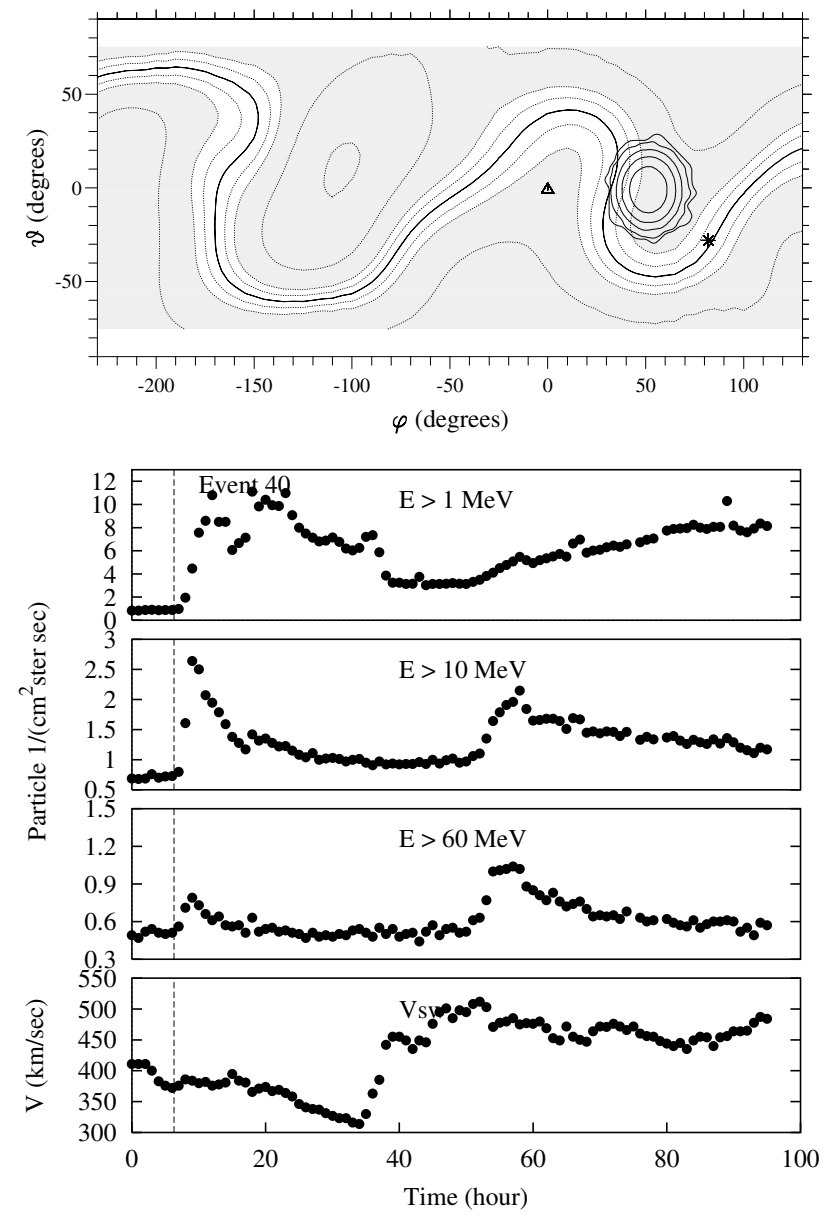

Fig. 4. Same as Fig. 1, but for event 40, on Nov. 22, 1998. Note that the solar magnetic configuration is considerably distorted as compared to Fig. 1, due to the approach of the solar maximum.

shock latitudinal extent that can be inferred from Fig. 2 of Dalla et al. (2003): in that figure, it can be seen that energetic particles reach the spacecraft when the latitudinal separation is less than about $20^{\circ}-25^{\circ}$. The agreement of the typical CME shock sizes obtained from different studies supports the interpretation of the SEP data in terms of shock acceleration.

On the other hand, several authors are questioning the "current paradigm" on the basis of the fact that SEPs at high heliographic latitudes are observed by Ulysses even in absence of shocks at high latitude, and with a considerable time delay with respect to the flare (Dalla et al. 2003; Zhang et al. 2003). The data analysis of those authors indicates that SEPs could propagate transverse to the magnetic field more than field line random walk. Also, Cane \& Erickson (2003) pointed out that in some cases SEPs are observed too early to be accelerated by a CME shock following the flare. All the above authors suggest enhanced particle propagation (over field line random walk) perpendicular to the average field so that the $30^{\circ}$ difference in longitude could be covered mostly by particle transport. Indeed, Cane \& Erickson (2003) suggest that, in the most energetic flares, particles can travel perpendicular to the average field up to $\pm 40^{\circ}$. This enhanced transport for particles can be obtained because of gyroresonant effects (especially for large gyroradii), and because particles can move back and forth along 
the magnetic field because of parallel scattering (Giacalone \& Jokipii 1999; Kota \& Jokipii 2000; Qin et al. 2002), and take advantage of the stochasticity of field lines (Rechester \& Rosenbluth 1978). However, a consensus on particle perpendicular transport has not yet been achieved (see Qin et al. 2002; Matthaeus et al. 2003, for a discussion).

A third possibility for longitudinal propagation is represented by the fact that different western longitudes for the Earth's magnetic foot point correspond to the slow wind and to the fast wind (about $60^{\circ} \mathrm{W}$ for the former and about $30^{\circ} \mathrm{W}$ for the latter). As a consequence, a strong magnetic shear is found at the boundary between fast and slow streams. Even if flare-accelerated particles start, say, in a slow stream, they can reach a fast stream by field line random walk in latitude, and propagate efficiently in longitude because of the different spiral angle in the fast stream. Zimbardo et al. (2001) and Pommois et al. (2002) have estimated that because of this effect the magnetic foot point of the Earth can correspond to an area as large as $30^{\circ}-40^{\circ}$ in longitude, if the Earth is located in a transition region from fast to slow wind. We also note that the longitudes of most of the flares for which particles are observed rage from W30 to W76, which is consistent with the foot point longitudes spanned by the magnetic shear of the solar wind, once the field line random walk $\left( \pm 10^{\circ}\right)$ is considered. This mechanism alone would not explain the observations of energetic particles associated with flares with either low western or eastern longitudes. On the other hand, for a numerical simulation aiming to a direct comparison with the SEP data, a detailed knowledge of the solar wind stream structure (as a funcion of $\vartheta$ and $\varphi$ ) is necessary.

Thus, we have studied the magnetic connection from the Earth to the solar corona, taking into account the observed magnetic turbulence level and the measured solar wind speed, for a set of 42 flares taken from the Goes X-rays catalogue for the period 1996-1998. We found that the magnetic foot area has a longitudinal half-width of $6^{\circ}-10^{\circ}$, and a latitudinal half-width of $10^{\circ}-15^{\circ}$, depending on the turbulence level. On the other hand, energetic particles from the Sun are observed even when the longitudinal separation of the flare and the nominal magnetic foot point is $25^{\circ}-30^{\circ}$. In order to fill this gap, we discussed three possibilities, namely the size of the flare-associated shock, particle perpendicular diffusion enhanced over field line diffusion, and the presence of magnetic shear in the magnetic structure of the solar wind. The relative contribution of each mechanism has to be assessed by case studies.

Acknowledgements. This work is part of a research programme which is financially supported by the Italian MIUR (Cofin 2002), by the Center for High Performance computing (Centro di Eccellenza MIUR) at the University of Calabria, and by the European Union Research Training Network "Turbulence in Space Plasmas". Numerical calculations have been performed in the framework of HPCC (Center for High Performance Computing) of the University of Calabria.

\section{References}

Bavassano, B., Dobrowolny, M., Fanfoni, G., et al. 1982, Sol. Phys., 78, 373

Belcher, J. W., \& Davis, L. 1971, J. Geophys. Res., 76, 3534

Cane, H. V., Reames, D. V., \& von Rosenvinge, T. T. 1988, J. Geophys. Res., 93, 9555

Cane, H. V., \& Erickson, W. C. 1971, J. Geophys. Res., 108, 1203

Dalla, S., Balogh, A., Krucker, S., et al. 2003, Geophys. Res. Lett., 30(19), 8035

Giacalone, J., \& Jokipii, J. R. 1999, ApJ, 520, 204

Gray, P. C., Pontius, D. H., \& Matthaeus, W. H. 1996, Geophys. Res. Lett., 23, 965

Hundhausen, A. J. 1993, J. Geophys. Res., 98, 13177

Jokipii, J. R., \& Parker, E. N. 1968, Phys. Rev. Lett., 21, 44

Jokipii, J. R., Kóta, J., Giacalone, J., et al. 1995, Geophys. Res. Lett., 22,3385

Kallenrode, M. B. 1993, J. Geophys. Res., 98, 5573

Kóta, J., \& Jokipii, J. R. 1995, Science, 268, 1024

Kóta, J., \& Jokipii, J. R. 2000, ApJ, 531, 1067

Matthaeus, W. H., Qin, G., Bieber, J. W., et al. 2003, ApJ, 590, L53

Pommois, P., Zimbardo, G., \& Veltri, P. 1998, Phys. Plasmas, 5, 1288

Pommois, P., Veltri, P., \& Zimbardo, G. 1999, Phys. Rev. E, 59, 2244

Pommois, P., Veltri, P., \& Zimbardo, G. 2001a, Phys. Rev. E, 63, 066405

Pommois, P., Veltri, P., \& Zimbardo, G. 2001b, Nonlinear Proc. Geophys., 8, 151

Pommois, P., Veltri, P., \& Zimbardo, G. 2001c, J. Geophys. Res., 106(A11), 24965

Pommois, P., Veltri, P., \& Zimbardo, G. 2002, Proc. Meet.: SOLSPA 2001 Euroconference: Solar Cycle and Space Weather, Vico Equense, Italy 25-29 September 2001, ESA SP-477, 305

Qin, G., Matthaeus, W. H., \& Bieber, J. W. 2002, ApJ, 578, L117

Reames, D. V. 1999, Space Sci. Rev., 90, 413

Rechester, A. B., \& Rosenbluth, M. N. 1978, Phys. Rev. Lett., 40, 38

Teufel, A., \& Schlickeiser, R. 2002, A\&A, 393, 703

Teufel, A., \& Schlickeiser, R. 2003, A\&A, 397, 15

Zhang, M., McKibben, R. B., Lopate, C., et al. 2003, J. Geophys. Res., 108,1154

Zimbardo, G., Veltri, P., Basile, G., et al. 1995, Phys. Plasmas, 2, 2653

Zimbardo, G., Veltri, P., \& Pommois, P. 2000, Phys. Rev. E, 61, 1940

Zimbardo, G., Pommois, P., \& Veltri, P. 2001, Ap\&SS, 277, 97

Zimbardo, G., Pommois, P., \& Veltri, P. 2004, J. Geophys. Res., 109, A02113 